\title{
Temperature-dependent magnetoresistance of magnetic tunnel junctions with ultraviolet light-assisted oxidized barriers
}

\author{
U. May, K. Samm, H. Kittur, J. Hauch, R. Calarco, U. Rüdiger, ${ }^{\text {a) }}$ and G. Güntherodt \\ II. Phys. Institut, RWTH Aachen, 52056 Aachen, Germany
}

(Received 2 October 2000; accepted for publication 9 February 2001)

\begin{abstract}
$\mathrm{Co}(10 \mathrm{~nm}) / \mathrm{AlO}_{x}$ (nominally $\left.2 \mathrm{~nm}\right) / \mathrm{Co}(20 \mathrm{~nm})$ tunnel junctions have been prepared under ultrahigh vacuum conditions applying a shadow mask technique. An ultraviolet light-assisted oxidation process of the $\mathrm{AlO}_{x}$ barrier has been optimized by in situ $\mathrm{x}$-ray photoelectron spectroscopy, in conjunction with temperature-dependent tunneling magnetoresistance measurements. Optimum-oxidized tunnel junctions show a magnetoresistance of $20 \%$ at $285 \mathrm{~K}$, and up to $38 \%$ at $100 \mathrm{~K}$. For under-oxidized samples, with a remaining Al layer between the Co bottom electrode and the $\mathrm{AlO}_{x}$ barrier, the tunneling magnetoresistance decreases more rapidly with increasing temperature than observed for the over-oxidized samples. The resistance $\times$ area product of optimum-oxidized tunneling junctions exhibits a minimum, and increases for under- and over-oxidized samples.
\end{abstract}

Tunnel junctions with two ferromagnetic electrodes separated by a thin insulating layer show large magnetoresistance at room temperature, making them promising candidates for magnetic random access memory devices, magnetic recording heads, and magnetic sensor applications. ${ }^{1-3}$ For all applications, a reproducible low resistance $\times$ area product $(R \times A)$ is essential. Strong efforts have been spent on reducing the $R \times A$ product by reducing the thickness of the typically used $\mathrm{AlO}_{x}$ insulating barrier $(<10 \AA)$. Not only does the thickness of the insulating barrier, but also the oxidation process itself, has a significant influence on the $R \times A$ product. ${ }^{4}$ Different oxidation processes have been investigated, and optimized, in order to evaluate the efficiency and reliability for large scale oxidation of the barrier material. ${ }^{5-9}$ Natural oxidation yields to the lowest $R \times A$ product, but the oxidation time as well as the high failure rate are detrimental to industrial applications. In comparison with a plasma oxidation process, ultraviolet (UV) light-assisted oxidation ${ }^{10,11}$ has its merits in a lower $R \times A$ product and a smaller bias dependence of the tunneling magnetoresistance (TMR). ${ }^{4}$ The ozone generation in the presence of UV light increases the oxidation rate, but contrary to plasma oxidation processes there are no ions of high kinetic energy generating defects within the barrier due to bombardment effects.

We discuss the optimization of the UV light-assisted oxidation process of $\mathrm{AlO}_{x}$ barriers of UHV prepared $\mathrm{Co}(10$ $\mathrm{nm}) / \mathrm{AlO}_{x}(2 \mathrm{~nm}) / \mathrm{Co}(20 \mathrm{~nm}) \mathrm{Si}(100)$ junctions by monitoring the process via $\mathrm{x}$-ray photoelectron spectroscopy (XPS) as well as the TMR and the temperature dependence of the junctions $R \times A$ product as a function of oxidation time.

The tunneling junctions have been fabricated in UHV (base pressure $p=8 \times 10^{-11}$ mbar) by shadow mask deposition using electron beam evaporation. The area of the bottom and top Co electrodes across the $\mathrm{AlO}_{x}$ barrier is $150 \mu \mathrm{m}$ $\times 150 \mu \mathrm{m}$. The $2 \mathrm{~nm}$ thick $\mathrm{Al}$ layers have been deposited by e-beam evaporation, followed by an in situ oxidation process

${ }^{a)}$ Electronic mail: ruediger@physik.rwth-aachen.de in an $\mathrm{O}_{2}$ atmosphere using a $15 \mathrm{~W}$ UV lamp inside the chamber. For XPS measurements, the monochromatized $\mathrm{Mg} \mathrm{K} \alpha$ emission line of $E=h \nu=1253.6 \mathrm{eV}$ has been employed in conjunction with a hemispherical photoelectron energy analyzer with an energy resolution of $\Delta E / E=2 \%$. Low bias dc resistance and the TMR measurements have been performed in a variable temperature high magnetic field cryostat.

Figures 1 and 2 show the XPS spectra of UHV prepared $\mathrm{Al}(2 \mathrm{~nm}) / \mathrm{Co}(20 \mathrm{~nm})$ double layers in the $\mathrm{Al} 2 s$ and $2 p$ core level region $(55-135 \mathrm{eV})$, as well as in the Co $2 p$ core level region $(760-810 \mathrm{eV})$ as function of oxidation time. A nonoxidized $\mathrm{Al}(2 \mathrm{~nm}) / \mathrm{Co}(20 \mathrm{~nm})$ double layer acts as reference for the XPS investigation of the oxidation process (Fig. 1). After 1 min of UV light-assisted oxidation the $\mathrm{Al} 2 s$ (117.2 $\mathrm{eV})$ and $2 p(72.2 \mathrm{eV})$ peaks are clearly shifted by approximately $2.1 \mathrm{eV}$. The presence of a double peak structure for both levels indicates that the Al layer is not fully oxidized. Nevertheless, most of the $\mathrm{Al}$ has been oxidized within the first $10 \mathrm{~min}$. After $60 \mathrm{~min}$ of oxidation the double-peak like

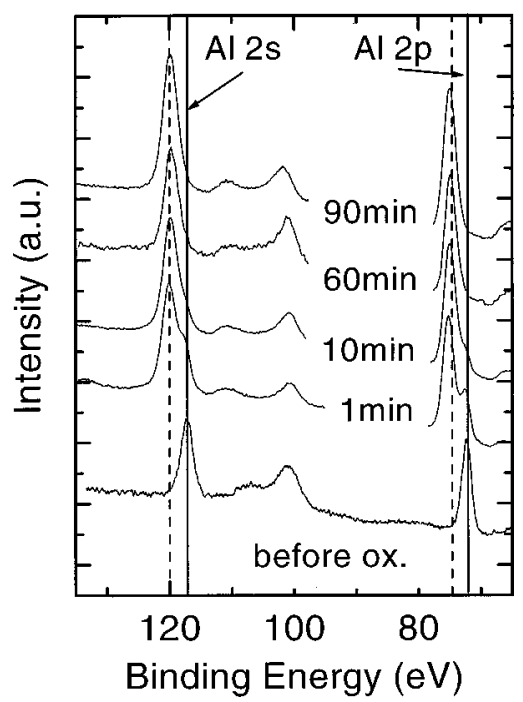

FIG. 1. XPS spectra of the $\mathrm{Al}(2 s$ and $2 p)$ core levels of $\mathrm{Al}(2 \mathrm{~nm}) / \mathrm{Co}(20$ $\mathrm{nm})$ double layers as a function of oxidation time $t_{\mathrm{ox}}$. 


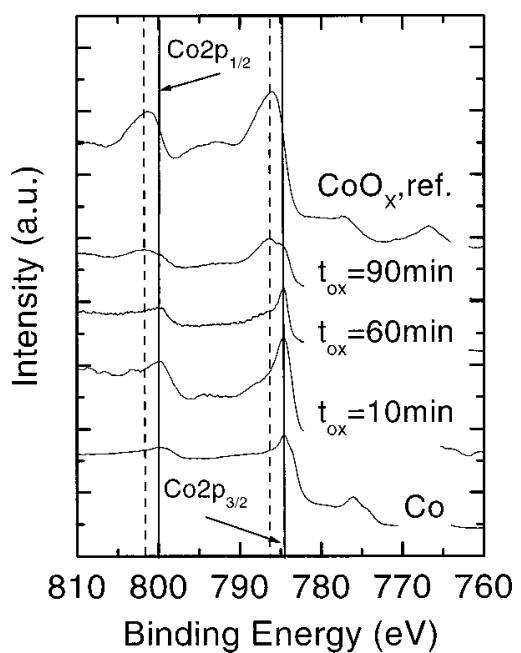

FIG. 2. XPS spectra of the Co $\left(2 p_{1 / 2}\right.$ and $\left.2 p_{3 / 2}\right)$ core levels of $\mathrm{Al}(2 \mathrm{~nm})$ / $\mathrm{Co}(20 \mathrm{~nm})$ double layers as function of oxidation time $t_{\mathrm{ox}}$.

structure of the $\mathrm{Al}$ core levels disappears, indicating a fully oxidized Al layer. For oxidation times larger than 60 min, the XPS spectra in the Al core level region does not change anymore.

The observed Co core level $\left(2 p_{1 / 2}\right.$ and $\left.2 p_{3 / 2}\right)$ spectra also support that the 60 min oxidation time is the optimum, achieving a fully oxidized Al layer without oxidizing the underlying Co electrode (Fig. 2). Starting from a Co XPS reference spectrum the peak positions of the Co core levels $\left[E\left(2 p_{1 / 2}\right)=799.5 \mathrm{eV}\right.$ and $\left.E\left(2 p_{3 / 2}\right)=784.4 \mathrm{eV}\right]$ stay unchanged up to an oxidation time of $60 \mathrm{~min}$. The XPS spectrum for an oxidation time of 90 min clearly shows the appearance of a double peak structure for both $2 p$ core levels, indicating the ongoing oxidation of the Co electrode. For the $\mathrm{CoO}_{x}$ reference sample a shift of the Co $2 p$ core levels by $1.6 \mathrm{eV}$ has been determined. From the XPS study one can conclude that an oxidation time of approximately $60 \mathrm{~min}$ leads to tunneling junctions with optimum tunneling barriers.

Figures 3(a) and 3(b) show the TMR values of $\operatorname{Co}(10$ $\mathrm{nm}) / \mathrm{AlO}_{x}(2 \mathrm{~nm}) / \mathrm{Co}(20 \mathrm{~nm})$ junctions as function of oxidation time at $T=100 \mathrm{~K}$ and $T=285 \mathrm{~K}$, respectively. At 100 $\mathrm{K}$ [Fig. 3 (a)] the TMR exhibits a sharp maximum of $36 \%$ for an oxidation time of $t_{\mathrm{ox}}=60 \mathrm{~min}$. Slightly under- and over-oxidized samples (45 and 75 min oxidation time) reveal a reduction of the TMR to approximately $20 \%$. The reduction of the TMR for under- and over- oxidized samples appears to be symmetric about the optimum time of $t_{\mathrm{ox}}=60$ min.

At $T=285 \mathrm{~K}$ the maximum TMR for $t_{\mathrm{ox}}=60 \mathrm{~min}$ is reduced to $20 \%$ [see Fig. 3(b)]. In contrast, the TMR values as a function of oxidation time are not symmetric about the optimum time. For under-oxidized samples $\left(t_{\mathrm{ox}} \leqslant 55 \mathrm{~min}\right)$ the TMR drops more rapidly than for the over-oxidized samples $\left(t_{\mathrm{ox}} \geqslant 65 \mathrm{~min}\right)$. For example the TMR for junctions with $t_{\mathrm{ox}}$ $=55 \mathrm{~min}$ has been determined to be $2.5 \%$, whereas the TMR for a sample with $t_{\mathrm{ox}}=65 \mathrm{~min}$ is still $15 \%$. Also modestly over-oxidized samples $\left(t_{\mathrm{ox}}=85 \mathrm{~min}\right)$ show a TMR of approximately $10 \%$.

The observed strong decrease of the TMR of underoxidized samples as a function of temperature can have different origins. First of all, one has to distinguish between the

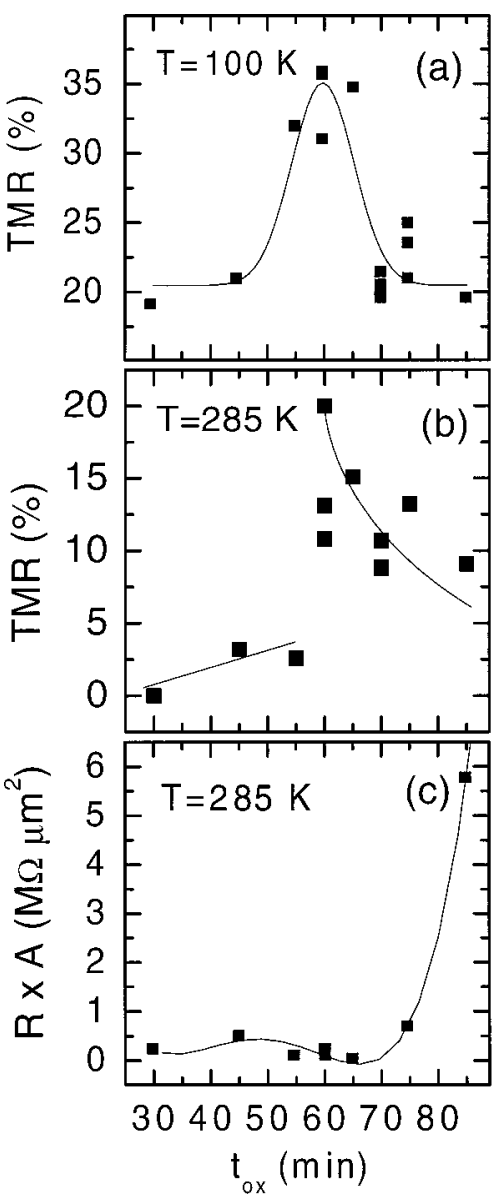

FIG. 3. Tunneling magnetoresistance of $\mathrm{Co}(10 \mathrm{~nm}) / \mathrm{AlO}_{x}(2 \mathrm{~nm}) / \mathrm{Co}(20 \mathrm{~nm})$ junctions as function of oxidation time at (a) $T=100 \mathrm{~K}$ and (b) $T=285 \mathrm{~K}$. (c) shows the $R \times A$ product of $\mathrm{Co}(10 \mathrm{~nm}) / \mathrm{AlO}_{x}(2 \mathrm{~nm}) / \mathrm{Co}(20 \mathrm{~nm})$ junctions as function of oxidation time at $T=285 \mathrm{~K}$.

temperature-dependence of the spin-dependent and spinindependent contribution to the resistivity. The decrease of the spin-dependent tunneling contribution is based on the reduction of the magnetization (spin-polarization) of the electrodes (especially at the electrode/barrier interface), due to the excitation of magnons and due to broadening of the Fermi distribution with increasing temperature. ${ }^{12}$ For the spin-independent contribution to the resistance a hopping process via localized states due to imperfections in the insulating barrier has been suggested. ${ }^{12}$ Such hopping processes can give rise to a temperature-dependent reduction of the TMR much faster than based on magnon excitations only.

In a simple model, under-oxidation leads to a very thin $\mathrm{Al}$ interlayer between the $\mathrm{AlO}_{x}$ and the Co bottom electrode or to $\mathrm{Al}$ inclusions within the already formed $\mathrm{AlO}_{x}$ barrier. In the presence of a nonuniformly oxidized barrier $\left(\mathrm{Al} / \mathrm{AlO}_{x}\right.$ double layer) or nonoxidized $\mathrm{Al}$ inclusions within the oxide barrier the temperature dependence of a spin-independent tunneling contribution can be larger than in the case of an over-oxidized sample with an additional $\mathrm{CoO}_{x}$ layer separating the Co bottom electrode and the $\mathrm{AlO}_{x}$ barrier.

On the other hand, a remaining Al layer on top of the Co bottom electrode can significantly reduce the surface spin polarization of the Co interface layer close to the Fermi energy $E_{F}$. Spin-polarized band structure calculations have shown that an $\mathrm{Al}$ termination of the Co bottom electrode strongly reduces the spin polarization of $\mathrm{Co}$ at the 


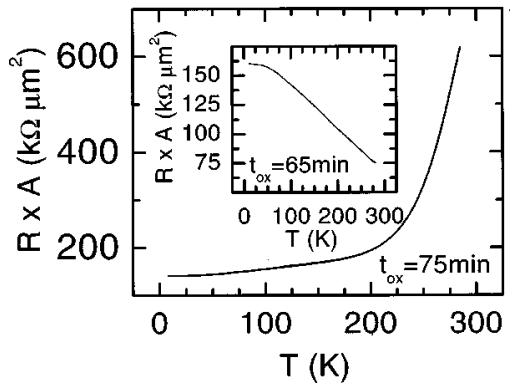

FIG. 4. The $R \times A$ product of an almost optimum-oxidized ( $t_{\mathrm{ox}}=65 \mathrm{~min}$ ) $\mathrm{Co}(10 \mathrm{~nm}) / \mathrm{AlO}_{x}(2 \mathrm{~nm}) / \mathrm{Co}(20 \mathrm{~nm})$ junction showing an insulator-like temperature dependence (inset). The $R \times A$ product of an over-oxidized $\left(t_{\mathrm{ox}}=75\right.$ min) nontunneling sample exhibits a metal-like temperature dependence.

interface. ${ }^{13,14}$ Also randomly distributed $\mathrm{Al}$ inclusions can have an influence on the spin polarization of the tunneling current. With an increasing impurity concentration within the barrier the spin polarization of the tunneling current decreases, leading to a reduction of the TMR..$^{15,16} \mathrm{~A}$ similar explanation has been given that Co can induce a spin polarization in the remaining $\mathrm{Al}$ layer which decreases with increasing Al layer thickness. ${ }^{17}$ Therefore, under-oxidation can affect the performance of junctions more seriously than overoxidation. But following these arguments it is unclear why a similar reduction of TMR at $T=100 \mathrm{~K}$ for under-oxidized samples has not been observed, or why the $\operatorname{TMR}\left(t_{\mathrm{ox}}\right)$ curve in Fig. 3(a) is symmetric.

The $R \times A$ at $T=285 \mathrm{~K}$ in Fig. 3(c) has a minimum of $158 \pm 64 \mathrm{k} \Omega \mu \mathrm{m}^{2}$ for an oxidation time of $t_{\mathrm{ox}}=65 \mathrm{~min}$. The observed decrease of the $R \times A$ product for a very short oxidation time $\left(t_{\mathrm{ox}}<45 \mathrm{~min}\right)$ is presumably due to the presence of highly conductive metallic pinholes through an incompletely formed $\mathrm{AlO}_{x}$ layer. The small maximum around $t_{\mathrm{ox}}=45 \mathrm{~min}$ is due to an enhanced density of defects and disorder in the under-oxidized barrier, as well as a not well defined $\mathrm{Co} / \mathrm{AlO}_{x}$ interlayer, which give rise to an enhanced resistance. The strong increase of the $R \times A$ product for an oxidation time longer than $75 \mathrm{~min}$ is given by a continuously increasing thickness of the $\mathrm{CoO}_{x} / \mathrm{AlO}_{x}$ barrier layer.

The inset of Fig. 4 shows the $R \times A$ product as function of temperature for an almost optimum-oxidized junction $\left(t_{\mathrm{ox}}=65 \mathrm{~min}\right)$ which decreases with increasing temperature as typical for tunneling junctions. In contrast, the $R \times A$ product of an over-oxidized sample showing no TMR $\left(t_{\mathrm{ox}}=75\right.$ $\mathrm{min}$ ) increases with increasing temperature pointing to metallic channels (pinholes) through the insulating barrier (see Fig. 4) ${ }^{18}$ Generally, over-oxidized samples showing no TMR can be divided into two different classes: one with a metal-like temperature dependence of the resistance $(R \times A$ product) due to the presence of highly conducting pinholes, and the other one with an insulator-like temperature dependence. In the latter case, the barrier consists of an insulating $\mathrm{CoO}_{x} / \mathrm{AlO}_{x}$ barrier as thick so that elastic spin-dependent tunneling processes are suppressed.

The determination of the TMR and the $R \times A$ product of $\mathrm{Co}(10 \mathrm{~nm}) / \mathrm{AlO}_{x}(2 \mathrm{~nm}) / \mathrm{Co}(20 \mathrm{~nm})$ junctions with UV lightassisted oxidized $\mathrm{AlO}_{x}$ barriers as a function of the oxidation time, offers a strategy for producing junctions with a satisfying large TMR value in conjunction with a small $R \times A$ product. The strong decrease of the TMR observed at $T=285$ $\mathrm{K}$ for under-oxidized samples with $t_{\mathrm{ox}} \leqslant 55$ min makes a reliable fabrication of optimum-oxidized tunneling devices hard to control. With reference to optimum-oxidized junctions, the slightly over-oxidized samples $\left(65 \mathrm{~min} \leqslant t_{\mathrm{ox}} \leqslant 75\right.$ min) reveal at $T=285 \mathrm{~K}$ a reduction of TMR of only 5 to $10 \%$, and the $R \times A$ product stays below $700 k \Omega \mu \mathrm{m}^{2}$.

A UV light-assisted oxidation process of $\mathrm{Co}(10 \mathrm{~nm}) /$ $\mathrm{AlO}_{x}(2 \mathrm{~nm}) / \mathrm{Co}(20 \mathrm{~nm})$ junctions has been investigated as a function of oxidation time. The TMR shows a sharp maximum as a function of oxidation time near $t_{\mathrm{ox}}=60 \mathrm{~min}$, but the decrease of TMR for over-oxidized junctions with increasing oxidation time is less pronounced than for underoxidized samples. For an "easy-to-control" fabrication process of tunneling junctions a slight over-oxidation seems to be favorable. In a further investigation, this oxidation strategy has to be applied for thinner $\mathrm{AlO}_{x}$ barriers.

This work was supported by the German Federal Ministry for Education and Research "BMBF" under Grant No. FKZ 13N7329, and the EC TMR Program "Submicron Magnetic Structures and Magneto-Transport Devices" (SUBMAGDEV).

${ }^{1}$ W. J. Gallagher, S. S. P. Parkin, Yu Lu, X. P. Bian, A. Marley, K. P. Roche, R. A. Altman, S. A. Rishton, C. Jahnes, T. M. Shaw, and G. Xiao, J. Appl. Phys. 81, 3741 (1997).

${ }^{2}$ J. S. Moodera, L. R. Kinder, T. M. Wong, and R. Meservey, Phys. Rev. Lett. 74, 3273 (1995).

${ }^{3}$ S. S. P. Parkin, K. P. Roche, M. G. Samant, P. M. Rice, R. B. Beyers, R. E. Scheuerlein, E. J. O‘Sullivan, S. L. Brown, J. Bucchigano, D. W. Abraham, Yu Lu, M. Rooks, P. L. Trouilloud, R. A. Wanner, and W. J. Gallagher, J. Appl. Phys. 85, 5828 (1999).

${ }^{4}$ H. Boeve, E. Girgis, J. Schelten, J. De Boeck, and G. Borghs, Appl. Phys. Lett. 76, 1048 (2000).

${ }^{5}$ H. Boeve, R. J. M. van de Veerdonk, B. Dutta, J. De Boeck, J. Moodera, and G. Borghs, J. Appl. Phys. 83, 6700 (1998).

${ }^{6}$ J. S. Moodera, L. R. Kinder, J. Nowak, P. LeClair, and R. Meservey, Appl. Phys. Lett. 69, 708 (1996).

${ }^{7}$ P. K. Wong, J. E. Evetts, and M. G. Blamire, J. Appl. Phys. 83, 6697 (1998).

${ }^{8}$ T. Miyazaki and N. Tezuka, J. Magn. Magn. Mater. 139, 1231 (1995).

${ }^{9}$ S. S. P. Parkin, R. E. Fontana, and A. C. Marley, J. Appl. Phys. 81, 5521 (1997).

${ }^{10}$ P. Rottländer, H. Kohlstedt, H. A. M. de Gronckel, E. Girgis, J. Schelten, and P. Grünberg, J. Magn. Magn. Mater. 210, 251 (2000).

${ }^{11}$ P. Rottländer, H. Kohlstedt, P. Grünberg, and E. Girgis, J. Appl. Phys. 87, 6067 (2000).

${ }^{12}$ C. H. Shang, J. Nowak, R. Jansen, and J. S. Moodera, Phys. Rev. B 58, R2917 (1998).

${ }^{13}$ I. Mertig, private communication.

${ }^{14}$ I. I. Oleinik, E. Y. Tsymbal, and D. G. Pettifor, Phys. Rev. B 62, 3952 (2000).

${ }^{15}$ E. Y. Tsymbal and D. G. Pettifor, Phys. Rev. B 58, 432 (1998).

${ }^{16}$ E. Y. Tsymbal and D. G. Pettifor, J. Appl. Phys. 85, 5801 (1999).

${ }^{17}$ J. S. Moodera, E. F. Gallagher, K. Robinson, and J. Nowak, Appl. Phys. Lett. 70, 3050 (1997).

${ }^{18}$ B. J. Jönsson-Ảkerman, R. Escudero, C. Leighton, S. Kim, I. K. Schuller, D. A. Rabson, Appl. Phys. Lett. 77, 1870 (2000). 